and thus our protocols seem to be safe. No healthcare workers in the clinic or close contacts of the patients became infected.

As SARS continues to spread worldwide, other healthcare settings will need to screen staff and patients who have symptoms and who have had close contact with SARS patients after an outbreak. ${ }^{13}$ With a sensitivity of $26 \%$ and a negative predictive value of $85 \%$, the WHO criteria should be refined to include routine daily follow up, documentation of nonrespiratory systemic symptoms, and daily chest radiography until patients have passed at least 48 hours without symptoms.

Contributors: THR had the idea for the study, oversaw its planning and execution and the statistical analysis, and prepared the manuscript. PAC, DS, and KLO participated in the planning, execution, and analysis. ANWH, DCPN, and ATA were responsible for assessment of radiographs and scans. LCYS planned the epidemiological follow up. JJYS supervised the clinical assessment of patients after admission. All authors contributed to the final version of the paper. THR will act as guarantor.

Funding: No additional funding.

Competing interests: None declared.

1 Poutanen SM, Low DE, Henry B, Finkelstein S, Rose D, Green K, et al. Identification of severe acute respiratory syndrome in Canada. $N$ Engl J Med 2003:348:1995-2005.
2 Tsang KW, Ho PL, Ooi GC, Yee WK, Wang T, Chan-Yeung M, et al. A cluster of cases of severe acute respiratory syndrome in Hong Kong. A cluster of cases of severe acu

3 Lee N, Hui D, Wu A, Chan P, Cameron P, Joynt G, et al. A major outbreak of severe acute respiratory syndrome (SARS) in Hong Kong. $N$ Engl J Med 2003;348:1986-94

4 Chan-Yeung M, Yu WC. Outbreak of severe acute respiratory syndrome in Hong Kong Special Administrative Region: case report. BMJ 2003;326:850-2.

5 Donnelly C, Ghani AC, Leung GM, Hedley AJ, Fraser C, Riley S, et al. Epidemiological determinants of spread of causal agent of severe acute respiratory syndrome. Lancet 2003;361:1761-6.

6 Peiris JSM, Chu CM, Cheng VCC, Chan KS, Hung IFN, Pool LLM, et al. Clinical progression and viral load in a community outbreak of coronavirus-associated SARS pneumonia: a prospective study. Lancet coronavirus-associ

2003;361:1767-72.
World Health Organization. Case definitions for surveillance of severe World Health Organization. Case definitions for surveillance of severe
acute respiratory syndrome (SARS). www.who.int/csr/sars/ casedefinition/en/ (accessed 10 April 2003).

8 Ho W, the Hong Kong Hospital Authority Working Group on SARS and the Central Committee on Infection Control. Guideline on managemen of severe acute respiratory syndrome (SARS). Lancet 2003;361:1313.

9 Peiris JSM, Lai ST, Poon LLM, Guan Y, Yam LYC, Lim W, et al. Coronavirus as a possible cause of severe acute respiratory syndrome. Lancet 2003:361:1319-25.

10 Ksiazek TG, Frdman D, Goldsmith C, Zaki SR, Peret T, Emery S, et al. A novel coronavirus associated with severe acute respiratory syndrome. New Engl J Med 2003;348:1953-66.

11 Drosten C, Gunther S, Preiser W, van der Werf S, Brodt H-R, Becker S, et al. Identification of a novel coronavirus in patients with severe acute respiratory syndrome. New EnglJ Med 2003;348:1967-76.

12 World Health Organization. Severe acute respiratory syndrome. $W k l y$ Epidemiol Rec 2003;78:97-120.

13 World Health Organization. Cumulative number of reported cases (SARS) from 1st November 2002 to 9th May 2003. www.who.int/csr/ sarscountry/2003 05 09/en/ (accessed 9 May 2003).

(Accepted 22 May 2003)
Department of Medicine and Therapeutics, Chinese University of Hong Kong, Prince of Wales Hospital, Shatin, New Territories, Hong Kong Special Administrative Region, China

Raymond S M Wong

haematologist

Alan Wu

medical and health

officer

Nelson Lee

medical and health

officer

David S Hui

associate professor

Gregory Cheng associate professor Joseph J Y Sung professor

continued over

BMJ 2003;326:1358-62

\title{
Haematological manifestations in patients with severe acute respiratory syndrome: retrospective analysis
}

Raymond S M Wong, Alan Wu, K F To, Nelson Lee, Christopher W K Lam, C K Wong, Paul K S Chan, Margaret H L Ng, L M Yu, David S Hui, John S Tam, Gregory Cheng, Joseph J Y Sung

\author{
Abstract \\ Objectives To evaluate the haematological findings \\ of patients with severe acute respiratory syndrome \\ (SARS). \\ Design Analysis of the demographic, clinical, and \\ laboratory characteristics of patients with SARS. \\ Setting Prince of Wales Hospital, Hong Kong. \\ Subjects All patients with a diagnosis of SARS \\ between 11 March and 29 March 2003 who had no \\ pre-existing haematological disorders. \\ Main outcome measures Clinical end points \\ included the need for intensive care and death. \\ Univariate and multivariate analyses were performed \\ to examine factors associated with adverse outcome. \\ Results 64 male and 93 female patients were included \\ in this study. The most common findings included \\ lymphopenia in 153 (98\%) of the 157 patients, \\ neutrophilia in $129(82 \%)$, thrombocytopenia in 87 \\ patients $(55 \%)$, followed by thrombocytosis in 77 \\ (49\%), and isolated prolonged activated partial \\ thromboplastin time in 96 patients $(63 \%)$. The \\ haemoglobin count dropped by more than $20 \mathrm{~g} / 1$ \\ from baseline in $95(61 \%)$ patients. Four patients \\ $(2.5 \%)$ developed disseminated intravascular \\ coagulation. Lymphopenia was shown in \\ haemato-lymphoid organs at postmortem
}

examination. Multivariate analysis showed that advanced age and a high concentration of lactate dehydrogenase at presentation were independent predictors of an adverse outcome. Subsets of peripheral blood lymphocytes were analysed in 31 patients. The counts of $\mathrm{CD} 4$ positive and $\mathrm{CD} 8$ positive $\mathrm{T}$ cells fell early in the course of illness. Low counts of CD4 and CD8 cells at presentation were associated with adverse outcomes.

Conclusions Abnormal haematological variables were common among patients with SARS. Lymphopenia and the depletion of T lymphocyte subsets may be associated with disease activity.

\section{Introduction}

An outbreak of severe acute respiratory syndrome (SARS) has recently been reported from Hong Kong. ${ }^{1}$ A novel coronavirus has been identified as the aetiological agent of the syndrome..$^{23}$ Viral infection may produce various haematological changes. Early studies have shown that lymphopenia and thrombocytopenia are common among patients with SARS. ${ }^{14}$ This study summarises the haematological findings in patients with SARS who were treated at the Prince of Wales Hospital, Hong Kong. 


\section{Methods}

\section{Study population}

Our study included all consecutive patients who received a diagnosis of SARS and were treated at the Prince of Wales Hospital but excluded patients with a history of haematological disorders. Based on the criteria for SARS that have been established by the US Centers for Disease Control and Prevention (CDC), our case definition was a fever (temperature $>38^{\circ} \mathrm{C}$ ), a chest radiograph or a computed tomographic image of the thorax showing evidence of consolidation with or without respiratory symptoms, and a history of close contact with a person in whom SARS had been diagnosed. The diagnosis was confirmed by an indirect immunofluorescence assay with fetal rhesus kidney cells that were infected with coronavirus and fixed in acetone to detect a serological response to the virus ${ }^{3}$ or by a positive viral culture.

\section{Laboratory studies}

Initial haematological investigations included a complete blood count with differential count and clotting profile (prothrombin time, activated partial thromboplastin time, and international normalised ratio). We studied these variables daily until a patient's fever had subsided for three days, then as clinically indicated, and at follow up visits after discharge from hospital. In some patients the chosen method was immunophenotyping with MultiTEST IMK kit, using flow cytometry (FACSCalibur 4 color system, BD Biosciences, California, United States) to obtain serial measurements of lymphocytes subsets in peripheral blood. We conducted postmortem investigations in some patients who died of SARS.

\section{Data collection and analysis}

We analysed the reported demographic, clinical, and laboratory characteristics. Day 1 was defined as the day of onset of fever. The clinical composite end point was the need for care in an intensive care unit, death, or both. We used univariate analysis to compare patients who reached the end point and those who did not, by using an unpaired Student's $t$ test, $\chi^{2}$ test, or Fisher's exact test, as appropriate. We then performed multiple logistic regression analysis, with stepwise analysis, to identify independent predictors of the end point after adjusting for age and concentration of lactate dehydrogenase at presentation. ${ }^{1}$ Only baseline variables were used as factors for predicting outcome, and those with a $\mathrm{P}$ value of less than 0.20 by univariate analysis were entered into the model. A $\mathrm{P}$ value of less than 0.05 was considered to indicate statistical significance. All probabilities are two tailed. We used SPSS for Windows (release 11.0.0) for statistical analysis. Data were reported as means (standard deviations) unless otherwise indicated.

\section{Results}

Between 11 and 29 March 2003 SARS was diagnosed in 160 patients at the Prince of Wales Hospital. We excluded three patients with a history of pancytopenia due to myelodysplastic syndrome $(\mathrm{n}=2)$ and systemic lupus erythematosus $(\mathrm{n}=1)$ from our analysis. We documented seroconversion or a greater than fourfold increase in antibody titre between acute and convalescence sera in 138 patients. Serum samples were not available in the remaining 19 convalescing patients, but six of them tested positive for coronavirus on culture. The study included 64 male and 93 female patients, all ethnic Chinese. Their mean age was 38.5 years (range 20-83 years). The median duration of follow up was 26 days (range 4-38 days). All patients received broad spectrum antibiotics and a combination of ribavirin and prednisolone $0.5 \mathrm{mg} / \mathrm{kg} /$ day as empirical treatment. Intravenous methylprednisolone at high dosage was used in patients with respiratory distress or progressive consolidations on their chest radiograph.

\section{White blood cells}

Transient leucopenia (leucocyte count $<4 \times 10^{9} /$ l) was found in $100(64 \%)$ of patients during their first week of illness. Ninety six patients $(61 \%)$ developed leucocytosis (leucocyte count $>11 \times 10^{9} / 1$ ), mostly during the second and third week of illness.

\section{Neutrophils}

Four patients $(2.5 \%)$ developed transient neutropenia (absolute neutrophil count $<0.5 \times 10^{9} /$ l) lasting for one to two days. We noted neutrophilia (absolute neutrophil count $\left.>7.5 \times 10^{9} / \mathrm{l}\right)$ in $129(82 \%)$ of patients. Univariate analysis showed that neutrophilia at any time was associated with a higher incidence of bacterial infections, documented by microbiological cultures $(\mathrm{P}=0.017)$. Most of these infections were hospital acquired pneumonia or line sepsis.

\section{Lymphocytes}

Lymphopenia (absolute lymphocyte count $<1000 /$ $\mathrm{mm}^{3}$ ) was noted in 153 (98\% of patients) during their course of illness. Most patients had a normal lymphocyte count at the onset of disease. Progressive lymphopenia occurred early in the course of illness and reached its lowest point in the second week in most cases. The lymphocyte count commonly recovered in the third week, but about 30\% (14 of 47) of patients remained lymphopenic at the fifth week of SARS (fig 1).

We analysed subsets of peripheral blood lymphocytes in 31 patients. The mean CD4 and CD8 cell counts at presentation were 286.7 (SD 142.2) cells $/ \mu \mathrm{l}$ (normal range 410 to 1590 cells $/ \mu \mathrm{l}$ ) and 242.2 (SD 130.8) cells $/ \mu \mathrm{l}$ (normal range 62 to 559 cells $/ \mu \mathrm{l}$ ) respectively. Most patients had reduced CD4 and CD8

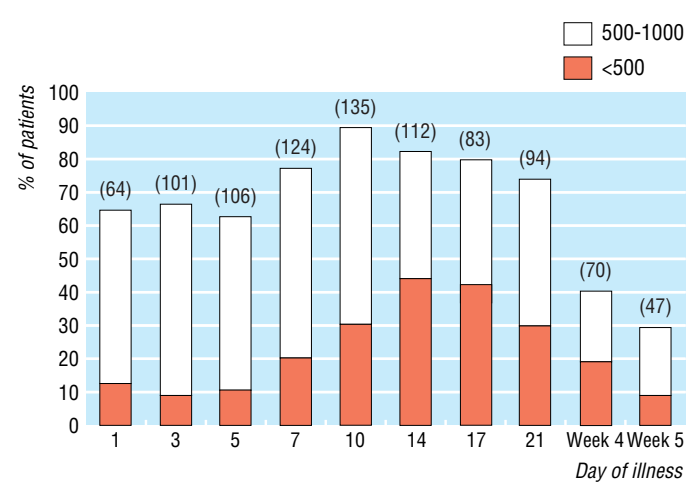

Fig 1 Proportion of patients with absolute lymphopenia during the course of SARS. The number in parentheses indicates the number of observations available at each of the different time points
Department of Anatomical and Cellular Pathology, Chinese University of Hong Kong

$\mathrm{K} \mathrm{F}$ To

associate professor

Margaret $\mathrm{H} \mathrm{L} \mathrm{Ng}$

associate professor

Department of

Chemical

Pathology, Chinese

University of Hong

Kong

Christopher W K

Lam

professor

C K Wong

associate professor

Department of

Microbiology,

Chinese University

of Hong Kong

Paul K S Chan

associate professor

John S Tam

professor

Centre for Clinical

Trials and

Epidemiological

Research, Chinese

University of Hong

Kong

$\mathrm{L}$ M Yu

statistician

Correspondence to:

JJ Y Sung

joesung@

cuhkeduhk 

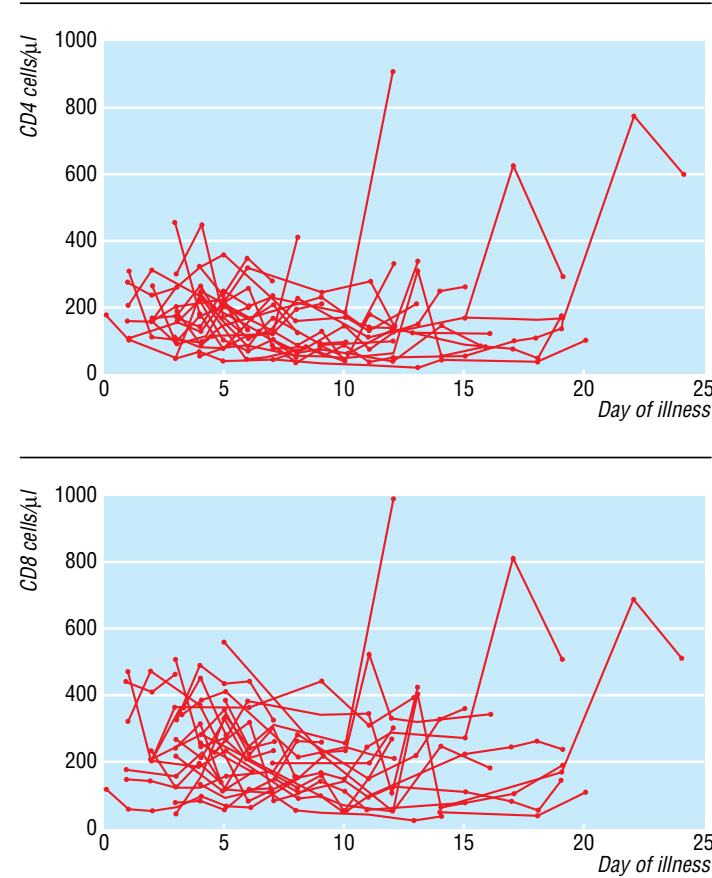

Fig 2 Individual plots of CD4 and CD8 lymphocyte subsets in peripheral blood against day of illness in 31 patients

cell counts during the early phase of illness, which reached a trough on days 5 to 14 and recovered gradually afterwards (fig 2). Five patients had an initial rise of CD4 and CD8 cell counts during the first week of illness, followed by a fall of both cell counts. The ratio of $\mathrm{CD} 4$ to $\mathrm{CD} 8$ cells remained in the normal range. The mean B lymphocyte count in peripheral blood at presentation was 151.3 (SD 73.2) per $\mu \mathrm{l}$ (normal range $90-660$ cells $/ \mu \mathrm{l})$ and remained stable.

\section{Platelet count}

Eighty seven patients $(55 \%)$ developed thrombocytopenia (platelet count $<140000 / \mathrm{mm}^{3}$ ) during the course of their illness. Most of them had mild thrombocytopenia, and only three (2\%) patients had a platelet count below $50000 / \mathrm{mm}^{3}$ (fig 1). Most patients' platelet count was normal at the onset of illness. Progressive thrombocytopenia occurred and reached a low point at the end of the first week. Thrombocytopenia was self limiting and resolved by the fourth week of illness. No patient had major bleeding or required platelet transfusion.

Reactive thrombocytosis (platelet count $\geq 400000 / \mathrm{mm}^{3}$ ) was noted in $77(49 \%)$ of patients (fig 3 ). The platelet count peaked on a median of day 17 of illness (range day 6 to 31). Only one patient had a platelet count above $1000000 / \mathrm{mm}^{3}$, and she had no evidence of thromboembolism.

\section{Haemoglobin}

Except for four patients with haemoglobin counts ranging from $95 \mathrm{~g} / 1$ to $105 \mathrm{~g} / \mathrm{l}$ due to $\beta$ thalassaemia trait $(n=1)$, iron deficiency $(n=2)$, and alcoholic liver disease $(n=1)$, all patients had normal haemoglobin counts at presentation. After two weeks of taking ribavirin at a dosage of $400 \mathrm{mg}$ every 8 hours intravenously or $1200 \mathrm{mg}$ thrice daily orally, the haemoglobin count dropped by more than $20 \mathrm{~g} / \mathrm{l}$ from baseline in 95 patients $(61 \%)$ and by more than $30 \mathrm{~g} / \mathrm{l}$ in 44 patients $(28 \%)$. We found no evidence of major bleeding, and no patient needed a transfusion or withdrawal of ribavirin. Of the 95 patients with a haemoglobin drop of more than $20 \mathrm{~g} / \mathrm{l}, 57$ showed evidence of haemolysis (rise of bilirubin $>20 \mu \mathrm{mol} / \mathrm{l}$ or reticulocyte count $>1 \%$, or both). We found no evidence of an immune related cause of haemolysis. Haemoglobin concentrations recovered gradually after treatment with ribavirin was stopped in all patients.

\section{Clotting profile}

We excluded from the analysis four patients with mechanical heart valve prostheses $(n=3)$ and atrial fibrillation $(\mathrm{n}=1)$ who were taking long term warfarin. Of the remaining 153 patients, 96 (63\%) had isolated prolonged activated partial thromboplastin time $(>40$ seconds) and normal prothrombin time during their course of illness (fig 4). The prolonged activated partial thromboplastin time ranged from 40.1 seconds to 68.1 seconds and occurred mainly in the first two weeks of illness. We found no evidence of venous thromboembolism or other coagulation abnormalities such as the presence of the anticardiolipin antibody or raised D-dimer concentrations. No patient required plasma transfusion or treatment with clotting factors. The abnormal clotting profile was self limiting, and all patients showed a normal activated partial thrombo-

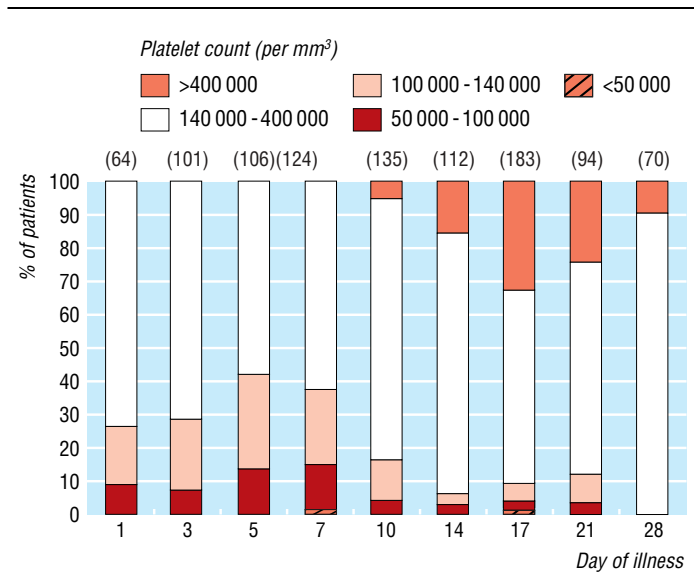

Fig 3 Proportion of patients with various platelet counts during the course of SARS. The number in parentheses indicates the number of observations available at each of the different time points

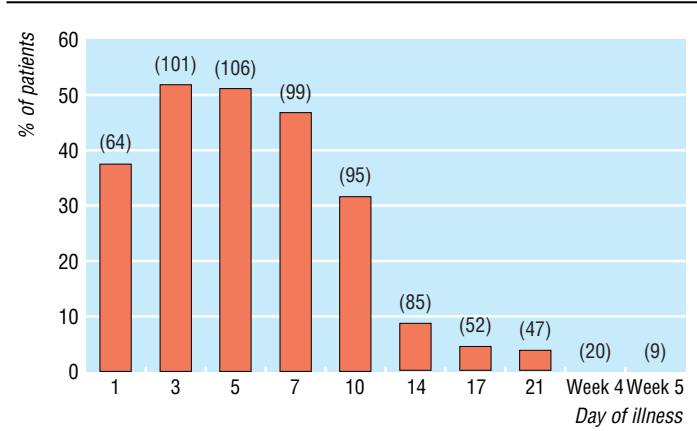

Fig 4 Proportion of patients with abnormal activated partial thromboplastin time during the course of SARS. The number in parentheses indicates the number of observations available at each of the different time points 
Univariate analysis of clinical and laboratory variables associated with the composite outcome of intensive care or death. Values are numbers $(\%)$ of patients with respective abnormalities unless otherwise indicated

\begin{tabular}{|c|c|c|c|c|}
\hline Variables & No intensive care & Intensive care or death & Odds ratio $(95 \% \mathrm{Cl})$ & $P$ value \\
\hline Platelet count $<140000$ per $\mathrm{mm}^{3}$ & $58 / 119(49)$ & $24 / 38(63)$ & 1.80 (0.85 to 3.82$)$ & $0.12^{*}$ \\
\hline Leucocyte count $>11 \times 10^{9} / \mathrm{l}$ & $61 / 119(51)$ & $15 / 38(39)$ & $0.62(0.30$ to 1.30$)$ & $0.21^{*}$ \\
\hline Mean (SD) absolute neutrophil count at presentation $\left(\times 10^{9} / \mathrm{l}\right)$ & $3.6(1.7)$ & $4.4 / 38(1.8)$ & 1.28 (1.04 to 1.57$)$ & $0.02 \dagger$ \\
\hline Absolute lymphocyte count $<1000$ per $\mathrm{mm}^{3}$ & 115/119 (97) & $37 / 38(97)$ & $1.29(0.14$ to 11.88$)$ & $1.00 \ddagger$ \\
\hline Development of disseminated intravascular coagulation§ & $0 / 118$ & $4 / 35(11.4)$ & & $0.05 \ddagger$ \\
\hline Isolated prolonged activated partial thromboplastin time & $76 / 118(64)$ & $22 / 35(63)$ & & $0.8^{*}$ \\
\hline Mean (SD) CD4 cell count at presentation $($ cells $/ \mu \mathrm{l})$ ף & $333.5(130.7)$ & $152.7(69.4)$ & & $0.029 \dagger$ \\
\hline 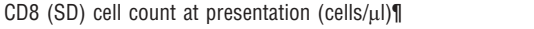 & $277.8(117.3)$ & $71.3(8.3)$ & & $0.006+$ \\
\hline
\end{tabular}

${ }^{*} \chi^{2}$ test.

tStudent's $t$ test.

$\ddagger$ Fisher's exact test.

$\S 0$ dds ratio cannot be calculated because the value in one of the cells was zero. १Analysis performed in a subgroup of 31 patients.

plastin time at week 4 . Fifty two patients (34\%) had normal clotting profiles throughout the course of their illness.

Four patients $(2.5 \%)$ developed frank disseminated intravascular coagulation with markedly prolonged prothrombin time, activated partial thromboplastin time, thrombocytopenia, and raised D-dimer concentrations. One patient had respiratory failure and needed mechanical ventilation at the time of analysis. The other three patients died of severe respiratory distress with multiorgan failure, and two of them had superimposed bacterial infections.

\section{Factors associated with admission to intensive care} and death

Univariate analysis showed that the development of thrombocytopenia and high absolute neutrophil count at presentation were associated with admission to intensive or death (table). In the multivariate analysis, advanced age and a high concentration of lactate dehydrogenase at presentation were independent predictors of adverse outcomes. In the subgroup of patients whose lymphocyte subsets were analysed, low CD4 and CD8 counts at presentation were associated with adverse outcomes.

\section{Haemato-lymphoid pathology}

At autopsy the haemato-lymphoid organs of the four patients who died showed no enlarged lymph nodes in peripheral soft tissues or other body parts. We noted no reactive lymphoid hyperplasia or $\mathrm{T}$ zone reaction. The splenic white pulps of all cases appeared atrophic with lymphoid depletion, and the red pulp was congested (fig 5). Bone marrow appeared active with presence of three lineages. We noted no features of hypoplastic marrow or reactive haemophagocytic syndrome in any of the patients. The pulmonary pathology was dominated by diffuse alveolar damage, and the lymphoid infiltrate was sparse. Viral isolation, in particular for coronavirus, showed negative results in the splenic, lymph node, and bone marrow tissues.

\section{Discussion}

Abnormal haematological variables were common among patients with the new respiratory illness known as SARS, which has had a large impact worldwide. ${ }^{5}$ Lymphopenia and the depletion of T lymphocyte subsets may be associated with disease activity.

Studies of its effect on various body systems are crucial to the understanding of this disease. Lympho- penia was the most common finding in our study in a cohort of 157 patients with SARS. Postmortem findings showed lymphopenia in various lymphoid organs. We found no features of bone marrow failure or reactive haemophagocytic syndrome. The inflammatory exudates in the lungs showed scanty lymphocytes. In those patients whose lymphocyte subset was analysed, both mean CD4 and CD8 cell counts were low at presentation and fell further during the early course of illness. The patients in this subgroup were young (median age 27 years, range 21 to 58 years) and had no comorbidity. We believe that the low CD4 and CD8 cell counts reflected the severity of infection due to the SARS virus and are therefore good markers of disease activity. Depletion of lymphocytes may be secondary to the direct effect of the virus on the lymphocytes or the effect of various cytokines. ${ }^{67}$ Neutrophilia was also common among patients with SARS and might be related to treatment with corticosteroids, but our results implied that full sepsis work up and empirical broad spectrum antibiotics should be considered in patients with neutrophilia.

Thrombocytopenia followed by reactive thrombocytosis was another common finding in our study. Thrombocytopenia has been reported in a variety of viral infections. ${ }^{8-13}$ It may be caused by an immune mechanism or a direct effect of viruses on megakaryocytes or platelets. ${ }^{13}$ Megakaryocytes are capable of harbouring a variety of viruses, although the mechanism of viral entry into megakaryocytes is not well understood. Dysmorphic megakaryocytes containing

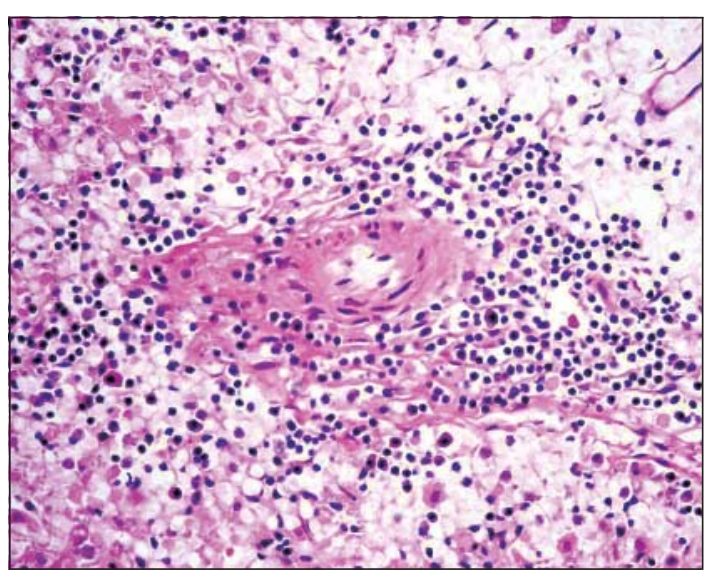

Fig 5 Histology of spleen. White pulp depletion was noted in all cases. Only a scanty amount of lymphoid cells were surrounding the splenic arterioles (haematoxylin and eosin stain, X200) 


\section{What is already known on this topic}

Severe acute respiratory syndrome (SARS) is an emerging infection that is spreading worldwide

The haematological features of this disease have not been described in detail elsewhere

Previous studies have indicated that patients with SARS may develop lymphopenia and thrombocytopenia, but the significance of these findings is uncertain

\section{What this study adds}

Lymphopenia was common among patients with SARS. It was also found in various lymphoid tissues on postmortem examination and may be a marker of disease activity

Both CD4 and CD8 counts decreased during the early course of SARS. Low CD4 and CD8 lymphocyte counts at presentation were associated with adverse outcomes

Leucocytosis with neutrophilia, thrombocytopenia, and isolated prolonged activated partial thromboplastin time were common in patients with SARS

inclusion bodies, vacuoles, degenerating nuclei, or showing naked nuclei may be seen in infected marrow. ${ }^{14}$ In most patients we found no evidence of disseminated intravascular coagulation. The postmortem findings of active bone marrow with normal megakaryoctes in patients with thrombocytopenia favour an immune cause of thrombocytopenia.

A major side effect of ribavirin is reversible haemolytic anaemia. ${ }^{15}{ }^{16}$ About $60 \%$ of our patients who received ribavirin experienced a drop of haemoglobin of more than $20 \mathrm{~g} / \mathrm{l}$. Despite this high drop, ribavirin was well tolerated and none of the patients needed withdrawal of treatment or transfusion. The development of anaemia may be a cause for concern. None the less haemolysis was transient, and haemoglobin counts improved after treatment with ribavirin had been completed in all cases. Careful monitoring of haemoglobin is advisable for patients who receive ribavirin.

\section{Conclusions}

Lymphopenia, in particular T lymphopenia, was common among patients with SARS in our study. A notable drop in CD4 and CD8 lymphocyte counts occurred early in the course of the syndrome and was associated with adverse outcomes. Thrombocytopenia, neutrophilia, and transient prolonged activated partial thromboplastin time were other common findings. Further studies to evaluate the mechanisms of these manifestations may help us to understand this disease.

We thank the Haematology Division of the Department of Anatomical and Cellular Pathology for their full support in the laboratory investigations.

Contributors: JJYS is guarantor and was responsible for patient management and coordinated the study. RSMW and GC were responsible for the study design, analysis, and writing of the paper. AW, NL, and DSH were responsible for patient management and data collection. KFT was responsible for postmortem examinations. MHLN, CWKL, and CKW were responsible for the haematological laboratory tests. PKSC and IST were responsible for the virological studies. LMY was responsible for the statistical analysis.

Funding: None

Competing interests: None declared.

Ethical approval: The study was approved by the ethics committee of the Department of Medicine and Therapeutics, Prince of Wales Hospital, Chinese University of Hong Kong.

1 Lee N, Hui D, Wu A, Chan P, Cameron P, Joynt GM, et al. A major outbreak of severe acute respiratory syndrome in Hong Kong. $N$ Engl Med 2003;348:1986-94

2 Ksiazek TG, Erdman D, Goldsmith CS, Zaki SR, Peret T, Emery S, et al. A novel coronavirus associated with severe acute respiratory syndrome. N Engl J Med 2003:348:1953-66.

3 Peiris J, Lai S, Poon L, Guan Y, Yam L, Lim W, et al. Coronavirus as a possible cause of severe acute respiratory syndrome. Lance 2003:361:1319-25.

4 Poutanen SM, Low DE, Henry B, Finkelstein S, Rose D, Green K, et al. Identification of severe acute respiratory syndrome in Canada. $N$ Engl J Med 2003;348:1995-2005.

5 Outbreak of severe acute respiratory syndrome--worldwide, 2003. MMWR Morb Mortal Wkly Rep 2003;52:226-8.

6 Maury CP, Lahdevirta J. Correlation of serum cytokine levels with haematological abnormalities in human immunodeficiency virus infection. J Intern Med 1990;227:253-7.

7 Van Campen H, Easterday BC, Hinshaw VS. Destruction of lymphocyte by a virulent avian influenza A virus. J Gen Virol 1989;70(Pt 2):467-72.

8 Eisenberg MJ, Kaplan B. Cytomegalovirus-induced thrombocytopenia in an immunocompetent adult. West J Med 1993;158:525-6.

9 Ninomiya N, Maeda T, Matsuda I. Thrombocytopenic purpura occurring during the early phase of a mumps infection. Helv Paediatr Acta 1977;32:87-9.

10 Oski FA, Naiman JL. Effect of live measles vaccine on the platelet count NEngl J Med 1966;275:352-6.

11 Van Spronsen DJ, Breed WP. Cytomegalovirus-induced thrombocytopenia and haemolysis in an immunocompetent adult. $\mathrm{Br} J$ Haemato 1996;92:218-20.

12 Ballem PJ, Belzberg A, Devine DV, Lyster D, Spruston B, Chambers H, et al. Kinetic studies of the mechanism of thrombocytopenia in patients with human immunodeficiency virus infection. $N$ Engl $J$ Med with human imm

13 Zucker-Franklin D. The effect of viral infections on platelets and megakaryocytes. Semin Hematol 1994;31:329-37.

14 Chesney PJ, Taher A, Gilbert EM, Shahidi NT. Intranuclear inclusions in megakaryocytes in congenital cytomegalovirus infection. J Pediatr 1978;92:957-8.

15 McCromick JB, King IJ, Webb PA, Scribner CL, Craven RB, Johnson KM, et al. Lassa fever. Effective therapy with ribavirin. $N$ Engl J Med 1986;314:20-6.

16 Roberts RB, Laskin OL, Laurence J, Scavuzzo D, Murray HW, Kim YT, et al. Ribavirin pharmacodynamics in high-risk patients for acquired immunodeficiency syndrome. Clin Pharmacol Ther 1987;42:365-73. (Accepted 23 May 2003)

\section{Corrections and clarifications}

Risk of subsequent thromboembolism for patients with pre-eclampsia

Sometimes an error persists beyond the $B M J$ and is noticed not by a reader or author but by one of our sister journals preparing the article for publication-in this case BMJ USA. In this Research Pointer by Carl van Walraven and colleagues (12 April, pp 791-2) we allowed a wrong confidence interval to slip through. In the last sentence in the first paragraph of the Methods section, the $95 \%$ confidence interval for the specificity (67\%) of the ICD-9 codes should be $59 \%$ to $74 \%$ [not $79 \%$ to $94 \%$ ].

ABC of antithrombotic therapy: Venous thromboembolism: treatment strategies

One of our eagle eyed readers alerted us to a surplus of zeros in this article by Alexander G G Turpie and colleagues (BMJ 2002;325:948-50). In the third sentence in the final paragraph on unfractionated heparin, the authors should have written the platelet count as $100 \times 10^{9} / 1$ [not $\left.100000 \times 10^{9} / 1\right]$. 